\title{
Male circumcision and penile cancer: a systematic review and meta-analysis
}

\author{
Natasha L. Larke $\cdot$ Sara L. Thomas • \\ Isabel dos Santos Silva $\cdot$ Helen A. Weiss
}

Received: 2 March 2011/ Accepted: 23 May 2011/Published online: 22 June 2011

(C) The Author(s) 2011. This article is published with open access at Springerlink.com

\begin{abstract}
Objective We systematically reviewed the evidence of an association between male circumcision and penile cancer. Methods Databases were searched using keywords and text terms for the epidemiology of penile cancer. Random effects meta-analyses were used to calculate summary odds ratios (ORs) and 95\% confidence intervals (CI).

Results We identified eight papers which evaluated the association of circumcision with penile cancer, of which seven were case-control studies. There was a strong protective effect of childhood/adolescent circumcision on invasive penile cancer $(\mathrm{OR}=0.33 ; 95 \%$ CI $0.13-0.83 ; 3$ studies). In two studies, the protective effect of childhood/ adolescent circumcision on invasive cancer no longer persisted when analyses were restricted to boys with no history of phimosis.
\end{abstract}

Electronic supplementary material The online version of this article (doi:10.1007/s10552-011-9785-9) contains supplementary material, which is available to authorized users.

N. L. Larke $(\bowtie) \cdot$ H. A. Weiss

MRC Tropical Epidemiology Group, Department of Infectious Disease Epidemiology, Faculty of Epidemiology and Population Health, London School of Hygiene and Tropical Medicine,

Keppel Street, London WC1E 7HT, UK

e-mail: Natasha.larke@1shtm.ac.uk

\section{S. L. Thomas}

Department of Infectious Disease Epidemiology, Faculty of Epidemiology and Population Health, London School of Hygiene and Tropical Medicine, Keppel Street, London WC1E 7HT, UK

\section{I. dos Santos Silva}

Department of Non-communicable Disease Epidemiology, Faculty of Epidemiology and Population Health, London School of Hygiene and Tropical Medicine, Keppel Street, London WC1E 7HT, UK
In contrast, there was some evidence that circumcision in adulthood was associated with an increased risk of invasive penile cancer (summary OR $=2.71 ; 95 \%$ CI $0.93-7.94 ; 3$ studies). There was little evidence for an association of penile intra-epithelial neoplasia and in situ penile cancer with circumcision performed at any age.

Conclusions Men circumcised in childhood/adolescence are at substantially reduced risk of invasive penile cancer, and this effect could be mediated partly through an effect on phimosis. Expansion of circumcision services in subSaharan Africa as an HIV prevention strategy may additionally reduce penile cancer risk.

Keywords Penile neoplasms - Male circumcision · Phimosis · Papillomavirus infections

\section{Introduction}

Penile cancer is relatively rare globally with an annual incidence of less than 1 case per 100,000 person-years (pyr) in Western countries [1]. However, recorded incidences are higher in many countries in sub-Saharan Africa including Uganda $(2.7 / 100,000 \mathrm{pyr})$, Swaziland (3.2/100,000 pyr) and Zimbabwe (1.6/100,000 pyr) [2-5]. Similarly, although penile cancer accounts for less than $0.5 \%$ of cancers in men globally, it accounts for a relatively higher proportion of cancers in Swaziland (4.4\%), Rwanda $(2.9 \%)$, Kenya (1.9\%) and Uganda $(1.7 \%)[2,3,6]$.

Penile cancer typically occurs in later life (median age at diagnosis in the United States of America (USA) is 68 years) [7]. Other factors associated with increased risk of penile cancer include low socio-economic status, cigarette smoking, human papillomavirus (HPV) infection, lack of penile hygiene, phimosis and penile inflammation [8]. 
The first suggestion of a link between circumcision and penile cancer was in 1932, when, among 1,103 penile cancer cases in the USA, none were Jewish despite $3 \%$ of the population being Jewish (and hence circumcised) [9]. Subsequent case series also showed a lower than expected prevalence of neonatal circumcision among penile cancer cases [10-12].

Ecological evidence came from studies in East Africa in the 1960s which showed that penile cancer was more common than expected in traditionally non-circumcising ethnic groups than among traditionally circumcising ethnic groups $[6,13]$. The first case-control study of penile cancer, published in 1947 among American military personnel, showed that circumcision in childhood was rare among cases of penile cancer compared to controls $(1.5 \%$ vs. $17.8 \%$; OR $=0.07,95 \%$ CI 0.01-0.28) [14].

Although male circumcision is widely cited to protect against penile cancer [15-19], the epidemiological evidence has never been systematically evaluated. Circumcision services are currently being expanded for HIV prevention in several African countries [20], and as penile cancer incidence is relatively high in many of these countries, expansion of circumcision services may provide an opportunity to reduce incidence of penile cancer.
The aim of this paper is to systematically review and summarise the epidemiological evidence of an association between male circumcision and penile intraepithelial neoplasia (PIN), in situ and invasive penile cancer, and to discuss the possible mechanisms and biological pathways for the association.

\section{Materials and methods}

\section{Search strategy}

Pubmed and Embase databases were searched on 22 April 2009 and updated on 15 September 2010 (for search terms see Box 1). No language restrictions were imposed. We did not include circumcision in the search terms to avoid detection bias. We also searched reference lists of relevant papers. Where information was missing from published papers, we contacted authors to obtain relevant information.

All abstracts were reviewed independently by two authors (NL and HW). Epidemiological studies likely to contain information on risk factors for penile cancer were deemed potentially relevant. Agreement about potential

Box 1 Search terms used in PubMed

\footnotetext{
Search 1

("Papillomavirus Infections/epidemiology" [MeSH] OR "Papillomavirus Infections/etiology"[MeSH] OR "Papillomavirus Infections/ prevention and control" [MeSH] OR "Papillomavirus Infections/transmission" [MeSH] OR "Papillomaviridae" [MeSH] OR "Condylomata Acuminata/epidemiology"[Mesh] OR "Condylomata Acuminata/etiology"[Mesh] OR "Condylomata Acuminata/prevention and control” [Mesh] OR “Condylomata Acuminata/transmission”[Mesh] OR "Warts/epidemiology”[Mesh] OR "Warts/etiology”[Mesh] OR "Warts/prevention and control"[Mesh] OR "Warts/transmission" [Mesh]OR wart*[text word] OR "papilloma virus"[text] OR "papilloma viruses"[text word] OR "HPV" [text] OR "condyloma acuminata"[text word] OR "condyloma acuminatum"[text word] OR papillomavir*[text word] OR “Penile Neoplasms/epidemiology”[Mesh] OR “Penile Neoplasms/etiology”[Mesh] OR "Penile Neoplasms/ prevention and control" [Mesh] OR "Penile Neoplasms/transmission" [Mesh] OR ((penil*[text] OR penis*[text]) AND (carcinoma*[text] OR cancer*[text] OR tumour [text] OR tumor [text] OR tumours[text] OR tumors[text] OR neoplas*[text] OR "Bowen's disease"[text] OR "Bowen disease"[text])) OR "Bowenoid Papulosis"[text] OR "Buschke-Lowenstein"[text] OR "Buschke Lowenstein"[text] OR “erythroplasia of Queyrat”[text]) AND ("Genital Diseases, Male/epidemiology”[Mesh] OR "Genital Diseases, Male/etiology”[Mesh] OR "Genital Diseases, Male/transmission" [Mesh] OR “Genital Diseases, Male/prevention and control”[Mesh] OR "Penile Diseases”[Mesh] OR “Sexually Transmitted Diseases, Viral/epidemiology"[Mesh] OR "Sexually Transmitted Diseases, Viral/etiology”[Mesh] OR "Sexually Transmitted Diseases, Viral/prevention and control” [Mesh] OR "Sexually Transmitted Diseases, Viral/transmission” [Mesh] OR "Genitalia, Male"[Mesh] OR Genital*[Text] OR penil*[text] OR penis*[text] OR scrot*[text] OR anogenital*[text] OR ano-genital*[text] OR urethra*[text] OR perine*[text]) AND ("Epidemiologic Studies"[MeSH] OR "Risk Factors"[MeSH] OR "Odds Ratio"[MeSH] OR "Prevalence" [MeSH] OR "Incidence" [MeSH] OR "logistic models" [MeSH] OR "multivariate analysis"[MeSH] OR prevalence[Text Word] OR incidence[Text Word] OR "odds ratio"[Text Word] OR "risk ratio" [Text Word] OR "risk factor"[Text Word] OR "relative risk" [Text Word] OR rate*[text]) AND ((Journal Article[pt] OR Letter[pt]) NOT "Case Reports" [Publication Type]) AND

"Humans"[MeSH Terms] AND ("Male"[MeSH] OR Male [Text] OR Males[Text])

Search 2 (excluding articles from search 1)

("Papillomavirus Infections/epidemiology" [MeSH] OR "Condylomata Acuminata/epidemiology"[Mesh] OR "Warts/epidemiology"[Mesh] OR "Penile Neoplasms/epidemiology"[Mesh]) AND ("Genital Diseases, Male/epidemiology"[Mesh] OR "Genital Diseases, Male/ etiology”[Mesh] OR “Genital Diseases, Male/transmission”[Mesh] OR “Genital Diseases, Male/prevention and control”[Mesh] OR "Penile Diseases”[Mesh] OR “Sexually Transmitted Diseases, Viral/epidemiology”[Mesh] OR "Sexually Transmitted Diseases, Viral/ etiology”[Mesh] OR "Sexually Transmitted Diseases, Viral/prevention and control”[Mesh] OR "Sexually Transmitted Diseases, Viral/ transmission" [Mesh] OR “Genitalia, Male"[Mesh] OR Genital*[Text] OR penil*[text] OR penis*[text] OR scrot*[text] OR anogenital*[text] OR ano-genital*[text] OR urethra*[text] OR perine*[text]) AND ((Journal Article[pt] OR Letter[pt]) NOT “Case Reports" [Publication Type]) AND "Humans"[MeSH Terms] AND ("Male"[MeSH] OR Male [Text] OR Males[Text])
} 
relevance was reached by consensus, and full text copies of those papers were obtained.

\section{Outcome definition}

Penile cancer included penile intra-epithelial neoplasia (PIN), in situ and invasive penile cancer. As in situ and invasive penile cancer may develop along different pathways $[21,22]$, and several studies have indicated that the effect of circumcision may vary depending on the stage of penile cancer [23-25], we decided a priori to analyse the effect of circumcision on (1) high-grade PIN and in situ penile cancer and (2) invasive penile cancer separately.

\section{Data extraction}

Data were extracted independently by two authors (NL and HW) using a standardised pre-piloted data extraction form. Inconsistencies were discussed by both reviewers and consensus reached.

Study populations described in more than one paper were included only once, using data from the paper with most information on the study methods.

\section{Methodological quality of included studies}

Seven quality domains were identified for case-control studies and six for cross-sectional studies (see supplementary material). We adopted an approach similar to that used by the Cochrane Collaboration for assessing risk of bias in randomised studies [26] and studies were classified as having either a high, low, or an unclear (where data were not reported) risk of bias for each quality domain. Specific criteria for assessing risk of bias are included in the supplementary material; of note where $20 \%$ or more identified prevalent cases could not be contacted, studies were considered to be at high risk of length bias (survival bias). This can occur when prevalent cases are missing because they are too ill to participate or have died, so the included cases represent those with less aggressive/advanced disease. If the exposure (i.e. circumcision) has a differential effect on different stages of disease then length bias may be introduced.

Two authors (NL and HW) reviewed the quality of individual studies, and inconsistencies were discussed by both reviewers and consensus reached.

\section{Data analysis}

The effect of circumcision on penile cancer was estimated using odds ratios (OR). Where ORs were not presented, crude ORs and 95\% CI were calculated from the data when possible, using exact methods. For each study, the effect estimate was calculated as the adjusted estimate where present and the crude estimate otherwise. This best available estimate was included in a random effects metaanalysis, using Stata 11. The meta-analysis included (1) analyses of circumcised men compared to men never circumcised, and (2) analyses in which the temporality between circumcision procedure and cancer diagnosis were defined (assuming that circumcision before the age of 18 years preceded penile cancer).

Circumcision at older ages may have been in response to penile cancer or pre-cancerous conditions, and a priori defined subgroup analyses were conducted to compare the association by age at circumcision.

Phimosis is a condition occurring most commonly in young boys where the foreskin cannot be fully retracted over the glans and is a risk factor for penile cancer $[8,23$, $24,27,28]$. Neonatal circumcision eliminates the risk of phimosis. Phimosis may be on the causal pathway between circumcision and penile cancer, so we defined a priori a subgroup analysis of the effect of circumcision on penile cancer among patients with no history of phimosis.

Summary estimates from sub-groups were formally compared using meta-regression. Heterogeneity was evaluated using the $I^{2}$ statistic and the $P$-value for heterogeneity [29]. The $I^{2}$ statistic represents the percentage of between-study variation that is due to heterogeneity rather than chance.

To address methodological concerns, we planned sensitivity analyses, which restricted analyses to: (1) studies which adjusted for covariates and (2) studies which ascertained circumcision status by clinical exam. However, due to the small number of eligible studies identified, only the sensitivity analysis of adjusted analyses with invasive penile cancer as the outcome could be conducted. For studies where age at circumcision was not adequately reported, sensitivity analyses were performed including them in meta-analyses of the most likely age at circumcision, based on information in the paper concerning the age at circumcision among cases or previously published data.

\section{Results}

Results of search strategy

A total of 3,366 papers were identified from the database searches and the full text was obtained for 306 of these (Fig. 1). A total of 269 papers were excluded based on the full text, including two studies that compared the prevalence of circumcision among cases of invasive penile carcinoma with cases of in situ penile cancer. An additional six papers were identified from references and review 


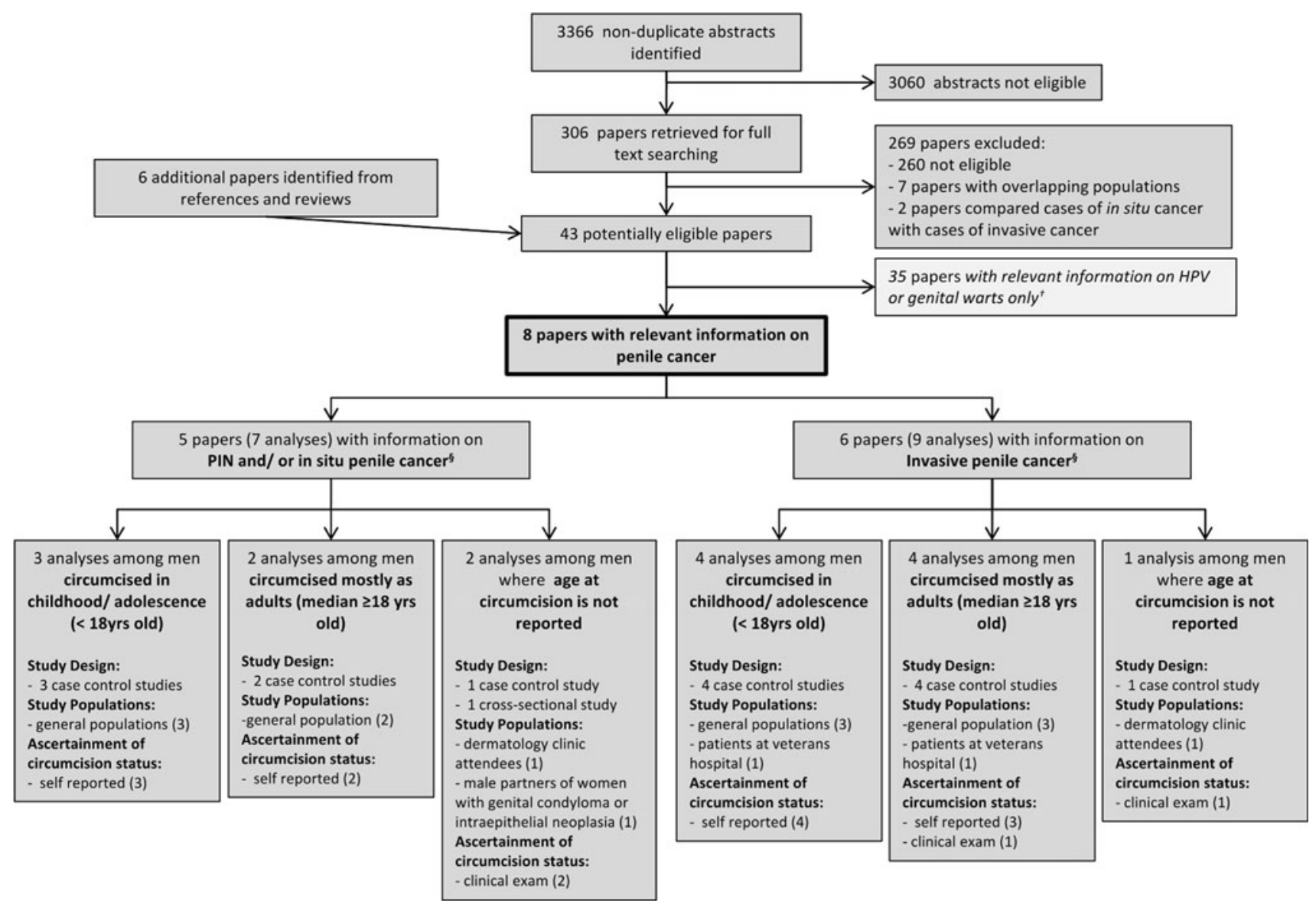

Fig. 1 Flow chart of study selection for inclusion in the systematic review. ${ }^{\S} 3$ papers evaluated the effect of circumcision on both in situ and invasive penile cancer. ${ }^{\dagger}$ The search was conducted as part of a

papers. Eight eligible papers were identified which included information on penile cancer.

\section{Description of eligible papers}

The eight papers evaluating the effect of circumcision on penile cancer included one cross-sectional study and seven case-control studies (Table 1). Study populations were from Europe $(n=4)$, the United States $(n=3)$, and China $(n=1)$.

Age at circumcision was categorised into three groups: childhood/adolescence (all participants $<18$ years); mostly adults (median age $\geq 18$ years; minimum 10 years old); and age inadequately reported (Table 1). The proportion of men circumcised during childhood/adolescence ranged from 4\% in Denmark [28] to $61 \%$ in the United States [23]. Circumcision among adults ranged from $0.7 \%$ in China [27] to 5\% in the USA [23].

Five papers evaluated the effect of circumcision on PIN and/or in situ cancer [23, 24, 28, 30,31] and six papers evaluated the effect on invasive cancer [14, 23, 24, 27, 28, 32] (Fig. 1; Table 1).

wider search strategy to identify papers on the association of male circumcision with HPV, genital warts and penile cancer

Quality of studies

One study had five quality domains classified as low risk and two domains classified as unclear risk [27]; all other studies had a high risk of bias in one or more of the quality domains (Table 2).

The potential for length bias was considered to be high for three case-control studies, where prevalent cases were identified and $\geq 20 \%$ could not be contacted for an interview [23, 24, 28] and unclear in one other study [14] (Table 2). For these studies, the proportion that were known to have died between diagnosis and the time of the study was 13\% [23], 17\% [24] and 34-38\% [28].

The risk of bias from poor participation was low for only one study [27] (Table 2). Fewer than $80 \%$ of identified subjects agreed to participate for three studies [23, 24, 28] and for one of these, the proportion that agreed to participate was substantially higher for controls $(60 \%)$ compared to cases $(27 \%)$ [28]. Information on participation was not available in three studies [30-32].

In four studies, the age at circumcision was not reported or incompletely reported, [24, 30-32] and the temporality 


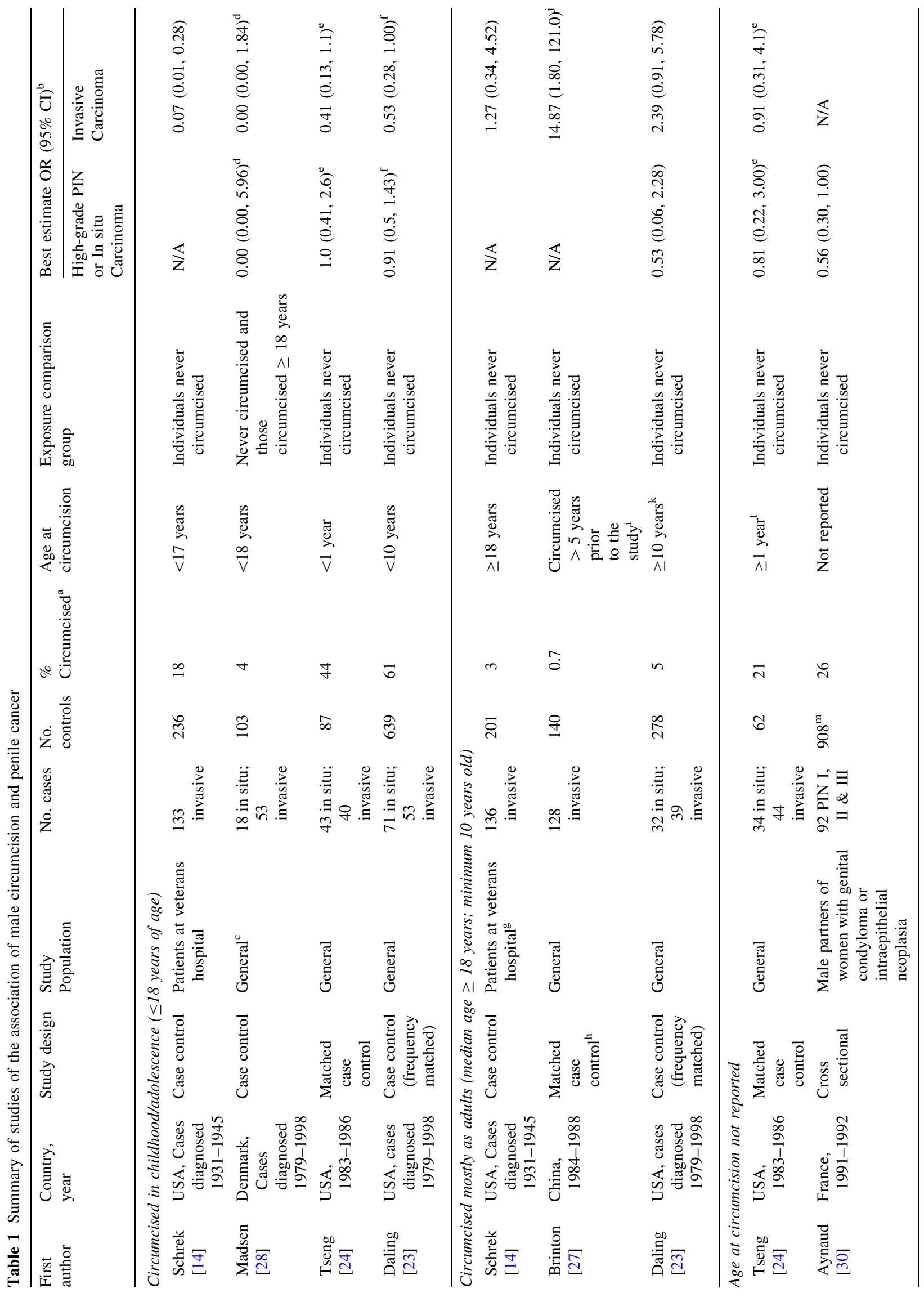




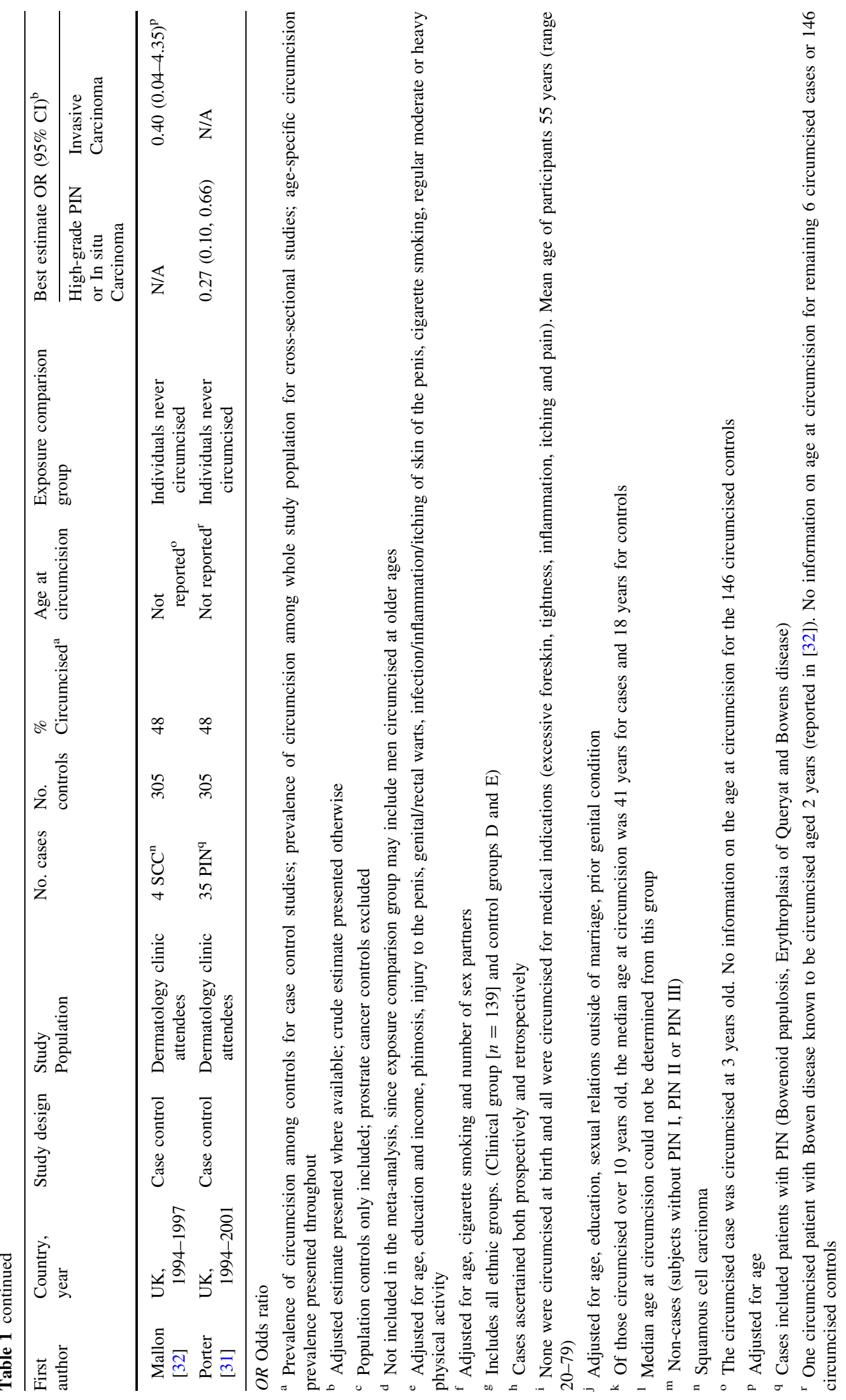




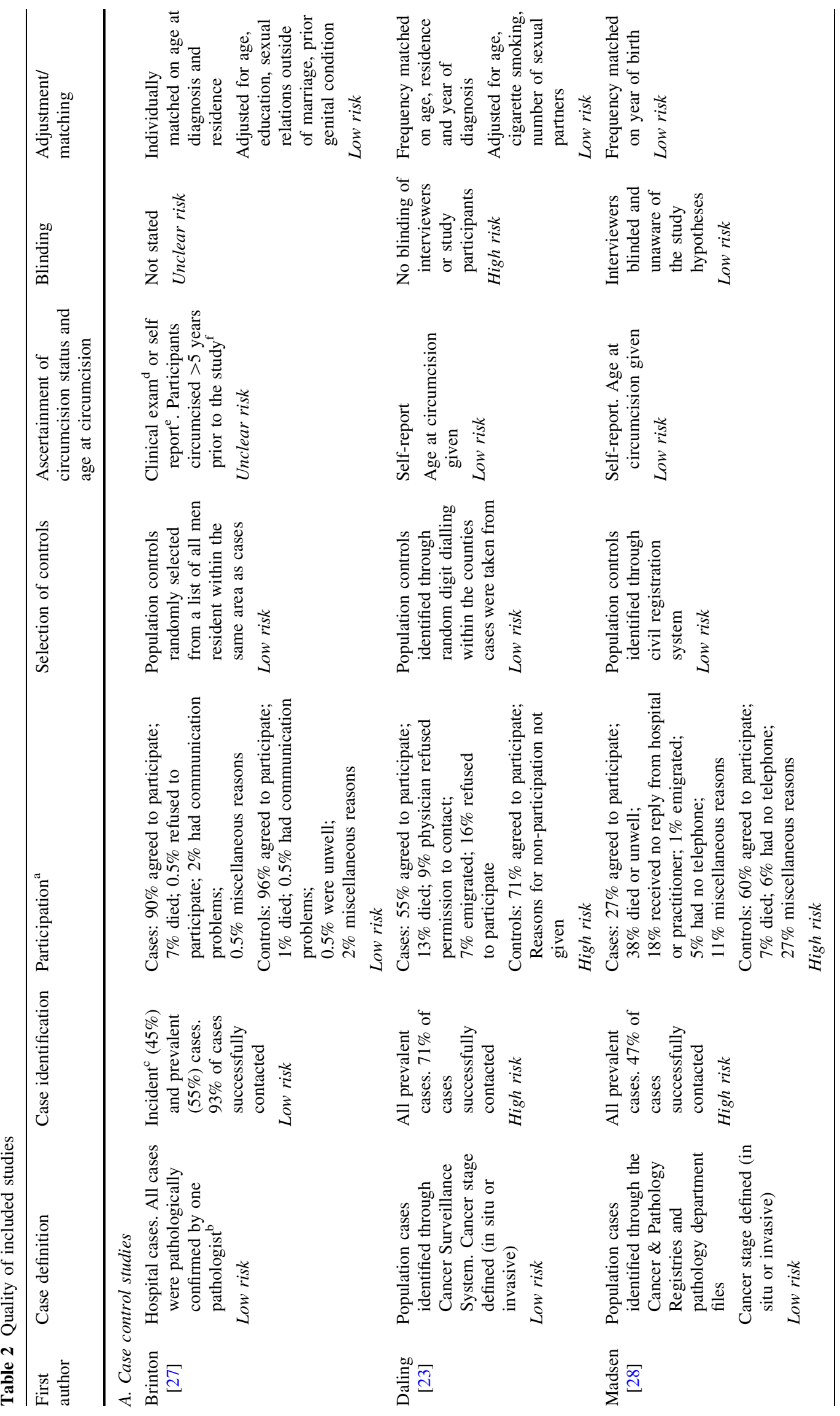




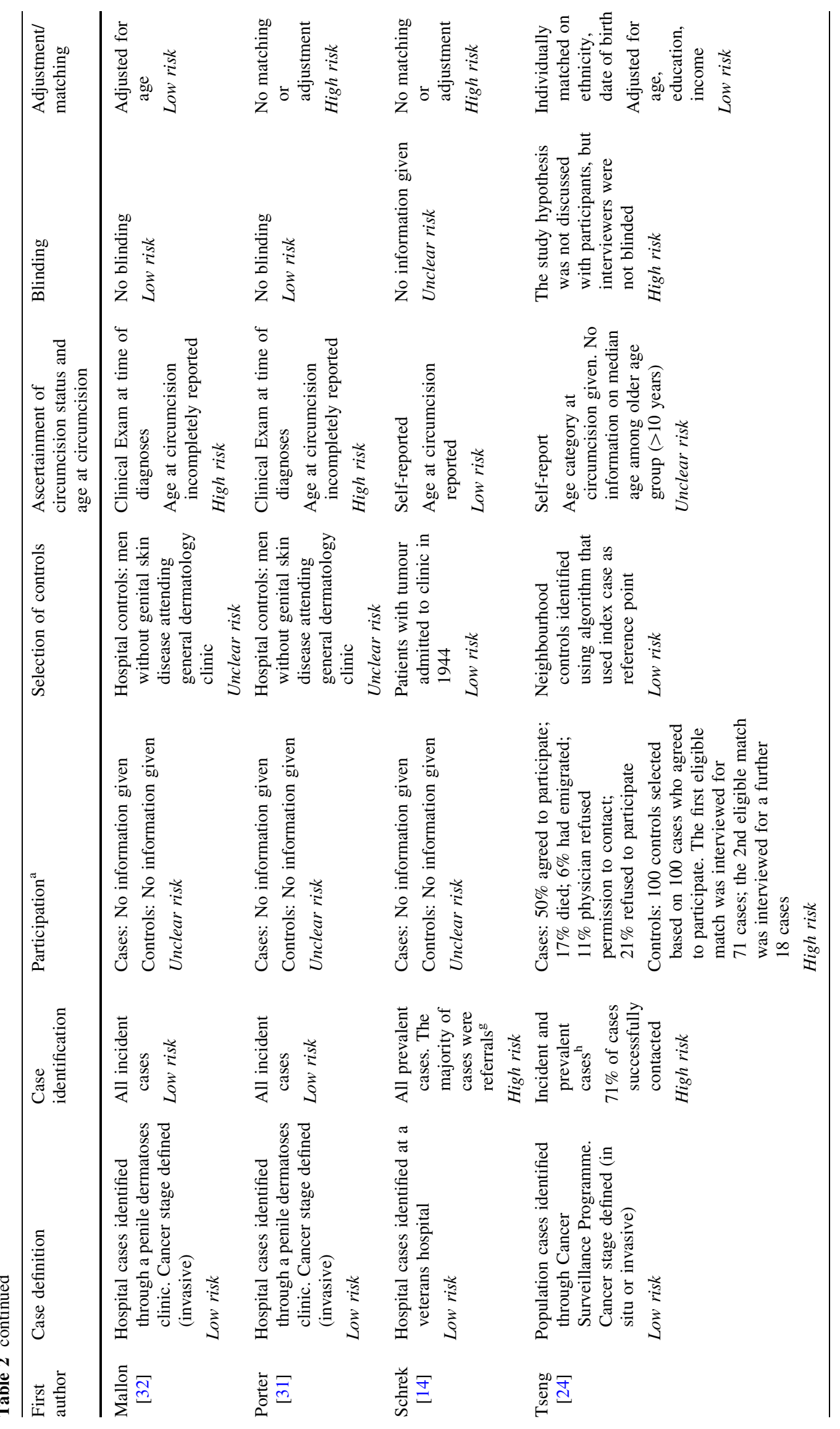




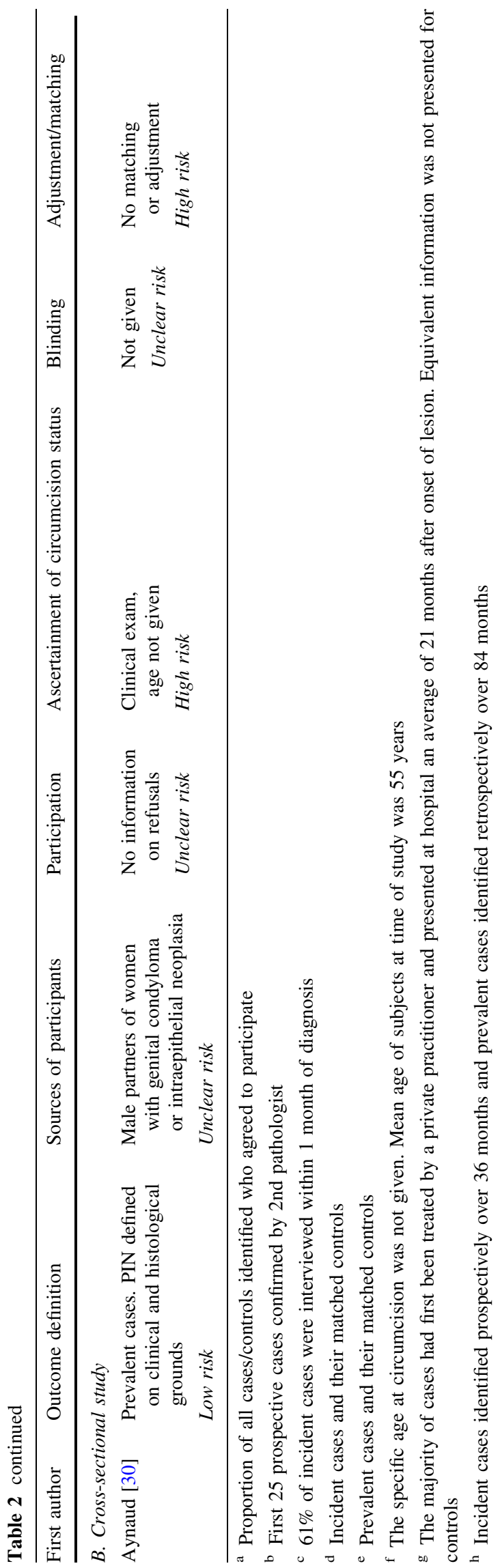

of any observed association between circumcision and penile cancer could not be assessed. In a fifth study [27], although the exact age at circumcision was not reported, the authors report that none of the boys were circumcised at birth and all participants were circumcised more than 5 years prior to the study. A high risk of confounding was present in three studies that did not adjust for age in either the study design or analysis [14, 30, 31].

Association between circumcision and penile cancer

Penile intra-epithelial neoplasia and in situ penile Cancer

Seven analyses evaluated the association of circumcision on PIN and in situ penile cancer [23, 24, 28, 30, 31] (Table 1; Fig. 1).

One of the three analyses of circumcision in childhood/ adolescence and PIN or in situ penile cancer compared men circumcised in childhood/adolescence with men not circumcised in childhood/adolescence [28] and was thus excluded from the meta-analysis. In this study, there were no penile cancer cases among men circumcised in childhood/adolescence, but the $95 \%$ CI was wide $(\mathrm{OR}=0.00$, 95\% CI 0.00-5.96). The remaining two analyses were deemed at low risk of bias from confounding in our quality assessment. These showed little evidence of an association of circumcision with PIN and in situ penile cancer (summary $\mathrm{OR}=0.93,95 \%$ CI $0.59-1.47 ; \quad p$-heterogeneity $=0.86 ; I^{2}=0.0 \%$ ) (Fig. 2). The OR from the single analysis among men circumcised mostly as adults was smaller $(\mathrm{OR}=0.53 ; 95 \%$ CI $0.06-2.28)$ but confidence intervals were wide, and there was little evidence of a difference in this effect estimate and the summary estimate for those circumcised in childhood/adolescence ( $p=0.60)$.

Three analyses did not adequately report age at circumcision. For one (a cross-sectional study from France), circumcision was likely to have occurred in childhood and this showed some evidence of a protective effect of circumcision on PIN and in situ penile cancer $(\mathrm{OR}=0.56$; 95\% CI 0.30-1.00) [30]. A sensitivity analysis including this analysis with the other analyses of childhood/adolescent circumcision provided only weak evidence of a protective effect on PIN/in situ penile cancer (summary $\mathrm{OR}=0.77 ; 95 \%$ CI $0.54-1.11 p$-heterogeneity $=0.42$; $I^{2}=0.0 \% ; n=3$ ).

\section{Invasive penile cancer}

Nine analyses from six papers evaluated the effect of circumcision on invasive penile cancer $[14,23,24,27,28$, 32]; (Table 1; Fig. 1). Three of the four analyses of circumcision in childhood/adolescence, used a comparison group of never circumcised men. The overall OR from 
Fig. 2 Association of male circumcision and PIN (I-III) or in situ penile carcinoma: random effects meta-analysis
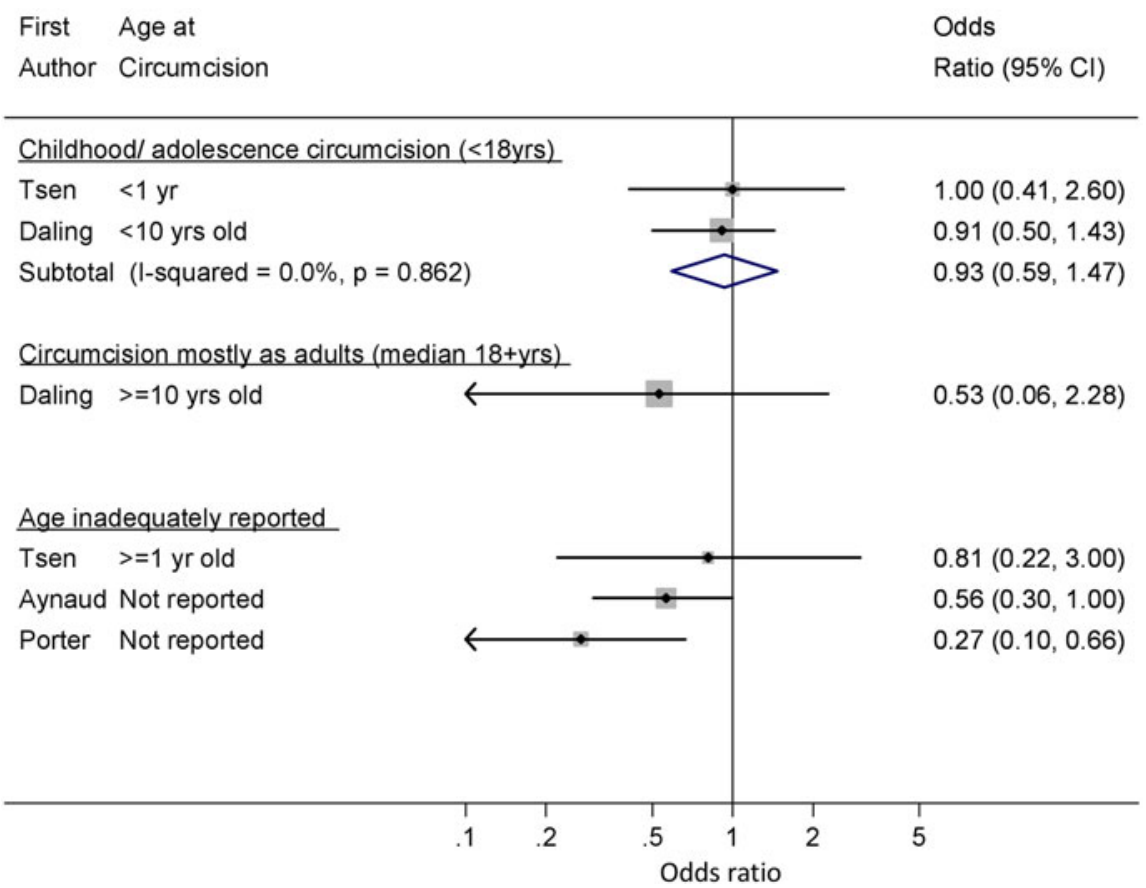

First

Author Age at Circumcision

Odds Ratio $(95 \% \mathrm{Cl})$

circumcision and invasive penile carcinoma: random effects meta-analysis

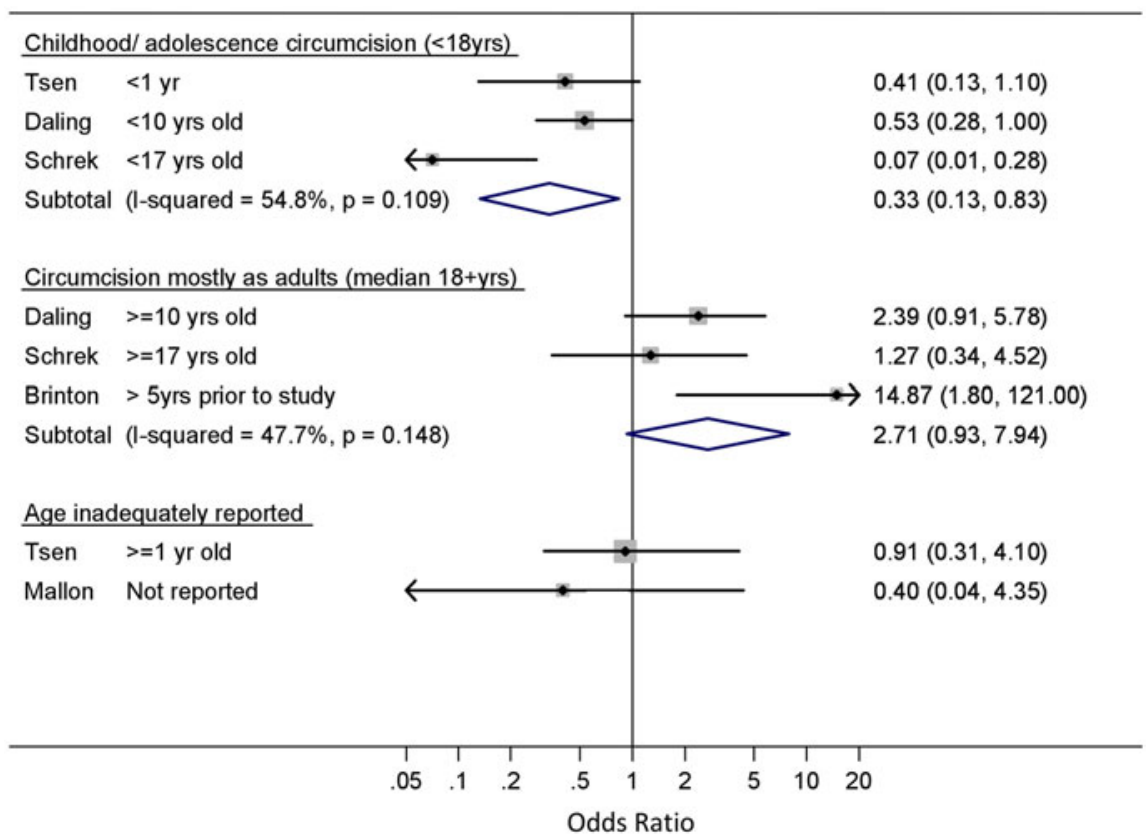

these three analyses showed strong evidence of a protective effect (summary OR $=0.33$; 95\% CI 0.13-0.83) (Fig. 3). There was some evidence of heterogeneity in estimates ( $p$-heterogeneity $\left.=0.11 ; I^{2}=55 \%\right)$ although all three studies showed a protective rather than detrimental effect. The sensitivity analysis including only the two analyses that adjusted for covariates showed little evidence of heterogeneity (summary OR $=0.50 ; 95 \%$ CI $0.29-0.86$; $p$-heterogeneity $\left.=0.69 ; I^{2}=0.0 \% ; n=2\right)$.

The relationship was reversed for analyses of men circumcised mostly as adults (summary OR $=2.71 ; 95 \% \mathrm{CI}$ $0.93-7.94 ; p$-heterogeneity $=0.15 ; I^{2}=48 \% ; 3$ analyses). 
Table 3 Effect of circumcision on invasive penile cancer by history of phimosis

\begin{tabular}{lllllc}
\hline First author & Study design & Age at circumcision & Comparison group & \multicolumn{2}{l}{ Adjusted OR (95\% CI) } \\
\cline { 4 - 7 } & & & All subjects & $\begin{array}{l}\text { Subjects with no history of } \\
\text { phimosis }\end{array}$ \\
\hline Daling [23] & Case control & $<10$ years & Not circumcised in childhood & $0.43(0.24,0.77)$ & $2.0(0.59,10.0)^{\mathrm{b}}$ \\
Tseng [24] & Matched case control & $<1$ year & Never circumcised & $0.41(0.13,1.1)$ & $0.79(0.29,2.6)^{\mathrm{c}}$
\end{tabular}

${ }^{a}$ Not circumcised in childhood $(<10$ years old) but those circumcised later in life are included

b A history of phimosis was only reported among those not circumcised in childhood ( $\geq 10$ years); among these children 254/278 controls had no history of phimosis and 20/39 cases of invasive cancer had no history of phimosis. Specific information on the numbers included in this analysis was not stated in the paper

c The analysis among those with no history of phimosis comprised all 44 participants who were circumcised at $<1$ year old and $61 / 83$ of the uncircumcised participants

Two analyses inadequately reported the age at circumcision and neither provided evidence of a protective effect of circumcision on invasive cancer (Table 1; Fig. 3). In one of these analyses, from a case-control study from the UK, the one cancer patient who was circumcised had been circumcised in childhood, and the controls are also likely to have been circumcised during childhood [32]. Including this analysis in the summary estimate for childhood/adolescent circumcisions gave similar results (summary $\mathrm{OR}=0.36 ; 95 \%$ CI $0.17-0.75 ; n=4)$ to the overall summary at these ages $(\mathrm{OR}=0.33 ; 95 \%$ CI $0.13-0.83$; $n=3$ ) with less heterogeneity in estimates ( $p$-heterogeneity $=0.22 ; \quad I^{2}=32 \% \quad$ vs. $\quad p$-heterogeneity $=0.11$; $I^{2}=55 \%$ ).

For the two analyses [23, 24] which assessed the effect of childhood/adolescent circumcision on both in situ and invasive penile cancer, there was a stronger association with invasive disease (OR 0.53 vs. 0.91 in the Daling et al. [23] paper and 0.41 vs. 1.0 in the Tseng et al. [24] paper) (Table 1). However, a meta-regression of the five analyses evaluating the effect of childhood/adolescent circumcision compared to no circumcision revealed weak evidence that the effect differed for in situ and invasive penile cancer $(p=0.20)$, although power for this analysis was low $[14,23,24]$.

\section{The role of phimosis in the association between circumcision and penile cancer}

To examine the association of circumcision with penile cancer beyond the effect mediated through phimosis, two papers stratified analyses by history of phimosis [23, 24]. Both of these found an association of circumcision with invasive penile cancer among all subjects, but no evidence of an association among those with no history of phimosis (Table 3).

\section{Discussion}

Circumcision in childhood/adolescence was protective against invasive penile cancer, with a summary odds ratio of 0.33 (95\% CI 0.13-0.83). Although there was some evidence of heterogeneity $\left(p=0.11, I^{2}=55 \%\right)$, all three studies demonstrated a protective effect (ORs 0.07, 0.41 and 0.53 , respectively). In contrast, there was some evidence that the risk of invasive penile cancer was elevated among men circumcised mostly as adults. There was little evidence of an association of circumcision at any age with PIN or in situ penile cancer.

\section{Quality of studies}

The overall risk of bias across studies was high, with only one study classified with no quality domains at high risk of bias [27]. Only two studies were confined to incident cases due to the rarity of penile cancer and in the studies which included at least some prevalent cases, risk of length (survival) bias was considered to be high. It is possible that the effect of circumcision may be different in the excluded cases; the effect of circumcision appears to be greater on invasive compared to PIN/in situ penile cancer and thus studies including prevalent cases could underestimate the true effect of circumcision on penile cancer. Specifically in analyses of childhood/adolescent circumcision and invasive cancer, all three included studies had a high risk of length bias. It is therefore possible that the summary odds ratio is an underestimate of the true effect.

Given the difficulties of conducting studies on penile cancer, the following could be considered in future studies to limit potential biases. Considering the rarity of penile cancer, multi-centre international case-control studies are the most feasible study design. However, prospectively ascertaining incident penile cancer cases to enrol in casecontrol studies will be the most effective method to limit 
length bias and could help to increase participation. Circumcision status should ideally be confirmed by a clinician. Finally, age at circumcision must be collected to allow the temporality between circumcision and penile cancer to be ascertained.

Effect of circumcision on in situ versus invasive penile cancer

Circumcision had a protective association with invasive penile cancer but there was no evidence of an effect on PIN/in situ. Two additional papers compared the prevalence of circumcision among cases of invasive penile cancer compared to in situ cancer [25, 33]. In one, the OR for invasive penile cancer (compared to in situ cancer) was 0.15 among men circumcised as newborns compared to uncircumcised men (95\% CI 0.02-0.66) [25]. In the second paper, none of the eight cases of invasive penile cancer were circumcised neonatally, whereas $46 \%$ of those cases with PIN or in situ penile cancer were circumcised neonatally; all eight cases (100\%) of invasive penile cancer were circumcised as adults and $8 \%$ of in situ cases were circumcised as adults [33].

There is evidence that some in situ cancers develop along a different pathway compared to invasive cancers $[21,22]$. PIN/in situ penile cancer is a diverse group of neoplasms ranging from the benign form of Bowenoid papulosis to the more aggressive forms of Bowen's disease and erythroplasia of Queyrat [34] and these may have differing potential to progress to invasive disease [35]. Similar observations have been made for vulvar cancer where two distinct aetiologic pathways have been described for basaloid/warty tumour and keratinizing tumours [36]. In one study, a strong effect was observed on erythroplasia of Queyrat (OR $=0.12,95 \%$ CI $0.00-0.90$, $n=315)$ and Bowenoid papulosis cases (OR $=0.20,95 \%$ CI 0.04-0.69), although there was no evidence of an effect on Bowen's disease (OR $=0.54,95 \%$ CI $0.01-10.58$, $n=308$ ) [31]. Considering the possibility of different developmental pathways for penile cancer, it is biologically plausible that circumcision has a different effect on specific aetiological subtypes.

Circumcision may also lead to increased detection of early stage penile cancers, for example if such tumours tend to be located under the foreskin. This increased ascertainment in circumcised men would mask a true underlying effect of circumcision on PIN/in situ penile cancer and may therefore explain the lack of effect on PIN/ in situ penile cancer observed in this review. Many penile tumours occur on the foreskin, and circumcision may exert an effect partially by removing a site susceptible to development of tumours.
Circumcision and phimosis

Phimosis is one of the strongest risk factors for penile cancer and four papers evaluated the association between phimosis and penile cancer (OR range 4.9-37.2 [23, 24, 27, $28]$ ). The effect of childhood/adolescent circumcision on invasive penile cancer may be largely mediated through elimination of phimosis, since there was no evidence of an association of circumcision with invasive disease when analyses were restricted to individuals with no history of phimosis [23, 24]. These results should be interpreted cautiously; they were secondary analyses among a subsample of the study populations and it is possible that they were underpowered. However, this is biologically plausible as circumcision eliminates risk of phimosis, and phimosis is likely to lead a build up of smegma and repeated inflammations, which may in turn lead to an increased risk of penile cancer [17]. Phimosis is a stronger risk factor for invasive disease compared to in situ cancer [23, 24] which further supports the argument that circumcision acts through prevention of phimosis and that some in situ cancers develop through a different pathway to invasive cancer.

Circumcision in adulthood

One possible reason for the increased risk of invasive penile cancer among men circumcised mostly as adults may be reverse causality: the surgery may have been performed as a treatment for penile cancer, a cancer precursor, or to treat an underlying medical condition known to be a risk factor for penile cancer (foreskin tightness, phimosis, or inflammation of the foreskin). Indeed in one study, all identified circumcisions were performed at older ages due to a medical indication, and although these were performed $>5$ years prior to the reference date [27], this may explain the significantly elevated risk for invasive cancer among circumcised men $(\mathrm{OR}=14.87)$.

\section{Circumcision and penile HPV infection}

Unlike cervical cancer, evidence suggests that HPV infection is not a necessary cause of penile cancer, with HPV prevalence ranging between 15 and $71 \%$ among penile cancer cases [21]. One paper measured HPV DNA in tumour tissue [23] and there was some suggestion of a greater protective association of childhood/adolescent circumcision with HPV negative tumours than HPV positive tumours although the CIs around these estimates were wide and overlapping $(\mathrm{OR}=0.48,95 \%$ CI $0.17-1.43$ vs. $\mathrm{OR}=0.83,95 \%$ CI $0.45-1.43)$. This association may be due to chance, and contrasts with evidence from systematic reviews [37] which showed circumcision is protective 
against HPV prevalence, and perhaps also against persistent [38] HPV infection in men. However, it may also reflect that HPV is more commonly associated with in situ tumours than invasive tumours [23], and the greater effect of circumcision on invasive rather than in situ tumours. An alternative explanation, is that HPV-PCR is compromised in penile cancer samples taken at later stages of disease due to tissue necrosis, and invasive penile cancer is in fact HPV-associated [39].

Potential impact in the scale up of circumcision services

In the two studies in the USA that calculated an adjusted OR, assuming that childhood/adolescent circumcision is causally related to invasive penile cancer, the population attributable fractions were $27 \%$ [23] and 50\% [24], suggesting that a considerable proportion of cases were due to a lack of circumcision in these populations. Circumcision prevalence is relatively high in the United States and it is quite likely a greater proportion of cases could be averted in countries with a lower prevalence of circumcision. For example in Swaziland, where circumcision is not commonly practised and penile cancer incidence is comparatively high (3.2 cases per 100,000 pyr [2, 3]), a national strategy exists to increase circumcision prevalence to $80 \%$ among HIV negative males aged 15-24 and to 33\% among male neonates as part of an HIV prevention strategy [40]. Successful implementation of this strategy may therefore lead to a considerable reduction in the incidence of penile cancer as well as HIV.

\section{Conclusions}

These data suggest that childhood/adolescent circumcision is protective against invasive penile cancer. This effect could be mediated partly through an effect on phimosis. Circumcision services among adults are currently being expanded as an HIV prevention strategy [20] and neonatal/ infant circumcision is emerging as a cost-effective longterm HIV prevention strategy [41]. Some countries in subSaharan Africa experience considerably higher incidence of penile cancer compared to Western countries and expansion of circumcision services in this region provides an opportunity to reduce penile cancer as well as HIV in men.

Acknowledgments We are grateful to the World Health Organisation for funding this research and providing funding for NL and ST. HW was funded by the UK Medical Research Council. We would also like to thank Chris Bunker, Janet Daling, Margaret Madeleine and HungFu Tseng for providing clarification of aspects of their studies.
Open Access This article is distributed under the terms of the Creative Commons Attribution Noncommercial License which permits any noncommercial use, distribution, and reproduction in any medium, provided the original author(s) and source are credited.

\section{References}

1. Parkin DM, Bray F (2006) Chapter 2: The burden of HPV-related cancers. Vaccine 24(Suppl 3):S3/11-25

2. Parkin DM (2002) Cancer incidence in five continents. International Agency for Research on Cancer, Lyon

3. Parkin DM, Ferlay J, Hamdi-Cherif M, Sitas F, Thomas JO, Wabinga $\mathrm{H}$ et al (2003) Cancer incidence in Africa. International Agency for Research on Cancer, Lyon

4. Wabinga HR, Parkin DM, Wabwire-Mangen F, Nambooze S (2000) Trends in cancer incidence in Kyadondo County, Uganda, 1960-1997. Br J Cancer 82(9):1585-1592

5. Parkin DM, Nambooze S, Wabwire-Mangen F, Wabinga HR (2010) Changing cancer incidence in Kampala, Uganda, 1991-2006. Int J Cancer 126(5):1187-1195

6. Dodge OG, Linsell CA, Davies JN (1963) Circumcision and the Incidence of Carcinoma of the Penis and the Cervix. A study in Kenya and Uganda Africans. East Afr Med J 40:440-444

7. Horner MJ, Ries LAG, Krapcho M, Neyman N, Aminou R, Howlader $\mathrm{N}$ et al. (2009) SEER cancer statistics review, 1975-2006

8. Wideroff L, Schottenfeld D (2006) Chapter 61. Penile cancer. In: Schottenfeld D, Fraumeni JF (eds) Cancer epidemiology and prevention. Oxford University Press, New York

9. Wolbarst A (1932) Circumcision and penile cancer. Lancet 219(5655):150-153

10. Dagher R, Selzer ML, Lapides J (1973) Carcinoma of the penis and the anti-circumcision crusade. J Urol 110(1):79-80

11. Jensen MO (1977) Cancer of the penis in Denmark 1942 to 1962 (511 cases). Dan Med Bull 24(2):66-72

12. Dean A (1935) Epithelioma of the penis. J Urol 33:252-283

13. Dodge O, Kaviti J (1965) Male circumcision among the peoples of East Africa and the incidence of genital cancer. East Afr Med J 42:99-105

14. Schrek R, Lenowitz H (1947) Etiologic factors in carcinoma of the penis. Cancer Res 7:180

15. Moses S, Bailey RC, Ronald AR (1998) Male circumcision: assessment of health benefits and risks. Sex Transm Infect 74(5):368-373

16. Schoen EJ (1991) The relationship between circumcision and cancer of the penis. CA Cancer J Clin 41(5):306-309

17. Misra S, Chaturvedi A, Misra NC (2004) Penile carcinoma: a challenge for the developing world. Lancet Oncol 5(4):240-247

18. Morris BJ (2007) Why circumcision is a biomedical imperative for the 21(st) century. Bioessays 29(11):1147-1158

19. Schoen EJ (1997) Benefits of newborn circumcision: is Europe ignoring medical evidence? Arch Dis Child 77(3):258-260

20. WHO/UNAIDS (2009) Progress in male circumcision scale-up: country implementation update. Accessed 9 March 2010; available from: www.malecircumcision.org

21. Rubin MA, Kleter B, Zhou M, Ayala G, Cubilla AL, Quint WG et al (2001) Detection and typing of human papillomavirus DNA in penile carcinoma: evidence for multiple independent pathways of penile carcinogenesis. Am J Pathol 159(4):1211-1218

22. Holly EA, Palefsky JM (1993) Factors related to risk of penile cancer: new evidence from a study in the Pacific Northwest. J Natl Cancer Inst 85(1):2-4

23. Daling JR, Madeleine MM, Johnson LG, Schwartz SM, Shera KA, Wurscher MA et al (2005) Penile cancer: importance of 
circumcision, human papillomavirus and smoking in in situ and invasive disease. Int J Cancer 116(4):606-616

24. Tseng HF, Morgenstern H, Mack T, Peters RK (2001) Risk factors for penile cancer: results of a population-based casecontrol study in Los Angeles County (United States). Cancer Causes Control 12(3):267-277

25. Schoen EJ, Oehrli M, Colby C, Machin G (2000) The highly protective effect of newborn circumcision against invasive penile cancer. Pediatrics 105(3):E36

26. The Cochrane Collaboration (2009) Cochrane handbook for systematic reviews of interventions

27. Brinton LA, Li JY, Rong SD, Huang S, Xiao BS, Shi BG et al (1991) Risk factors for penile cancer: results from a case-control study in China. Int J Cancer 47(4):504-509

28. Madsen BS, van den Brule AJ, Jensen HL, Wohlfahrt J, Frisch M (2008) Risk factors for squamous cell carcinoma of the penispopulation-based case-control study in Denmark. Cancer Epidemiol Biomarkers Prev 17(10):2683-2691

29. Higgins JP, Thompson SG, Deeks JJ, Altman DG (2003) Measuring inconsistency in meta-analyses. BMJ 327(7414):557-560

30. Aynaud O, Ionesco M, Barrasso R (1994) Penile intraepithelial neoplasia. Specific clinical features correlate with histologic and virologic findings. Cancer 74(6):1762-1767

31. Porter WM, Francis N, Hawkins D, Dinneen M, Bunker CB (2002) Penile intraepithelial neoplasia: clinical spectrum and treatment of 35 cases. Br J Dermatol 147(6):1159-1165

32. Mallon E, Hawkins D, Dinneen M, Francics N, Fearfield L, Newson R et al (2000) Circumcision and genital dermatoses. Arch Dermatol 136(3):350-354
33. Malek RS, Goellner JR, Smith TF, Espy MJ, Cupp MR (1993) Human papillomavirus infection and intraepithelial, in situ, and invasive carcinoma of penis. Urology 42(2):159-170

34. Gerber GS (1994) Carcinoma in situ of the penis. J Urol 151(4):829-833

35. Patterson JW, Kao GF, Graham JH, Helwig EB (1986) Bowenoid papulosis. A clinicopathologic study with ultrastructural observations. Cancer 57(4):823-836

36. Kurman RJ, Toki T, Schiffman MH (1993) Basaloid and warty carcinomas of the vulva. Distinctive types of squamous cell carcinoma frequently associated with human papillomaviruses. Am J Surg Pathol 17(2):133-145

37. Bosch FX, Albero G, Castellsague X (2009) Male circumcision, human papillomavirus and cervical cancer: from evidence to intervention. J Fam Plann Reprod Health Care 35(1):5-7

38. Gray RH, Serwadda D, Kong X, Makumbi F, Kigozi G, Gravitt $\mathrm{PE}$ et al (2010) Male circumcision decreases acquisition and increases clearance of high risk human papillomavirus in HIVnegative men: a randomized trial in Rakai, Uganda. J Infect Dis 201(10):1455-1462

39. Partridge JM, Koutsky LA (2006) Genital human papillomavirus infection in men. Lancet Infect Dis 6(1):21-31

40. Swaziland Male Circumcision Task Force (2009) Strategy and implementation plan for scaling up safe male circumcision for HIV Prevention in Swaziland

41. Binagwaho A, Pegurri E, Muita J, Bertozzi S (2010) Male circumcision at different ages in Rwanda: a cost-effectiveness study. PLoS Med 7(1):e1000211 